\title{
A NOTE ON THE RUIN PROBLEM FOR A CLASS OF STOCHASTIC PROCESSES WITH INTERCHANGEABLE INCREMENTS
}

\author{
Jan Grandell and Lars Peiram \\ University of Stockholm
}

\section{SUMmary}

Models for the risk business of an insurance company are often constructed by weighting pure Poisson models. In this paper it is verified that it is possible to calculate the probability of ruin in such weighted models by weighting ruin probabilities of pure Poisson models.

\section{INTRODUCTION}

In this paper we are going to study a model for the risk business of an insurance company where the claims are located according to a stochastic process $\{N(t) ; 0 \leqslant t<\infty\}$ subordinated to the Poisson process with a directing process $\{\Lambda(t) ; 0 \leqslant t<\infty\}$. This terminology follows Feller [3]. The directing process $\Lambda(t)$ will be a real-valued and non-decreasing process such that $P(\Lambda(\mathrm{o})=\mathrm{o})=\mathrm{r}$. We will later assume that $\Lambda(t)$ has stationary, and independent increments. Let $\{M(\mathrm{t}) ; 0 \leqslant \mathrm{t}<\infty\}$ be a Poisson process with intensity I, i.e.

$$
P(M(t)=k)=\frac{t^{k}}{k !} e^{-t}, k=0, \mathrm{I}, 2, \ldots
$$

The process $N(t)$ is then defined by $N(t)=M(\Lambda(t))$.

For each claim the company has to pay a certain amount counted with its proper sign. As usually in the theory of risk these amounts are assumed to be described by a sequence $\left\{X_{n}\right\}_{1}^{\infty}$ of independent random variables, each having the distribution function $V(x)$. These variables are further assumed to be independent of the process $\dot{N}(t)$.

The stochastic process 


$$
X(t)=\sum_{k=1}^{N(t)} X_{k} \quad(X(t)=0 \text { if } N(t)=0)
$$

will thus serve as a model for the total amount of claims paid by the company up to time $t$.

Looking a bit more formally upon this definition we let the stochastic vector process $\left\{\left(\Lambda(t), M(s), X_{k}\right) ; 0 \leqslant t, s<\infty, k=\right.$ $=\mathrm{I}, 2, \ldots\}$ be defined on $(\Omega, \mathfrak{X}, \Pi \mathfrak{X})$ where $\Omega$ is a sample space, $\mathfrak{X}$ the $\sigma$-algebra of subsets of $\Omega$ generated by the process and $\Pi \mathfrak{X}$ a probability measure on $\mathfrak{X}$. Let further $\mathfrak{L}, \mathfrak{M}, \mathfrak{B}$ and $\mathfrak{N}$ be the sub$\sigma$-algebras of $\mathfrak{X}$ generated by $\{\Lambda(t) ; 0 \leqslant t<\infty\},\{M(t) ; 0 \leqslant t<\infty\}$ $\left\{X_{k} ; k=\mathrm{I}, 2, \ldots\right\}$ and $\{(\Lambda(t), M(s)) ; 0 \leqslant t, s<\infty\}$ respectively and $\Pi_{\mathfrak{L}}, \Pi_{\mathfrak{M}}, \Pi_{\mathfrak{B}}$ and $\Pi_{\mathfrak{N}}$ the corresponding marginal measures.

For every $L \varepsilon \mathfrak{Q}, \quad M \varepsilon \mathfrak{M}$ and $V \varepsilon \mathfrak{B}$ we have $\Pi_{\mathfrak{N}}(L \cap M)=$ $=\Pi_{\mathfrak{R}}(L) . \Pi_{\mathfrak{M}}(M)$ and $\Pi_{\mathfrak{X}}(L \cap M \cap V)=\Pi_{\mathfrak{N}}(L \cap M) \cdot \Pi_{\mathfrak{B}}(V)$ because of the independence assumptions.

We will always consider only the separable version of the processes and further all measures will be assumed to be complete.

Let $\mathfrak{N}_{0}$ and $\mathfrak{X}_{0}$ be the $\sigma$-algebras generated by the above defined processes $\{N(t) ; 0 \leqslant t<\infty\}$ and $\{X(t) ; 0 \leqslant t<\infty\}$ respectively. Let $S$ be a countable but dense set in $[0, \infty)$. Because of the separability assumption $\{N(t)=k\}={ }_{S}^{U}\{M(s)=k, \Lambda(t)=s\}$. Thus $\mathfrak{R}_{0} \mathrm{C} \mathfrak{R}$. In the same way it is shown that $\mathfrak{X}_{0} \mathrm{CX}$.

\section{The case of a DiRecting process With STATIONARY AND INDEPENDENT INCREMENTS}

It is shown in Feller [3] that for every non-decreasing process $\Lambda(t)$ with stationary and independent increments (s.i.i. process)

where

$$
E e^{i u \Lambda(t)}=e^{t \eta(u)}
$$

$$
\eta(u)=\int_{0+}^{\infty} \frac{e^{i u x}-\mathrm{I}}{x} \Omega(d x)+i b u
$$

where $b \geqslant 0$ and $\int_{0}^{\infty} \frac{\Omega(d x)}{\mathrm{I}+x}<\infty$

The behaviour of the sample functions of non-decreasing s.i.i. processes is investigated by Walldin [5].

Since $M(t)$ is a s.i.i. process it follows from Feller [3] that also $N(t)$ is a s.i.i. process. 
For $N(t)$ we thus have

$$
\log E e^{i u N(t)}=t \cdot \sum_{k=1}^{\infty}\left(\mathrm{e}^{i u k}-\mathrm{I}\right) c_{k}
$$

where $c_{k} \geqslant 0$ for all $k$ and $\sum_{k=1}^{\infty} c_{k}<\infty$.

$N(t)$ is thus a bunch Poisson (Poisson par grappes) process. The bunches are located according to a Poisson process with intensity $c=\sum_{1}^{\infty} c_{k}$ and the distribution of the size of the bunches is given by $\frac{c_{k}}{c}$ for $k=\mathrm{I}, 2,3, \ldots$

\section{Theorem}

The relation between the measure $\Omega$ and the sequence $\left\{c_{k}\right\}_{1}^{\infty}$ is given by

$$
c_{k}=\int_{0+}^{\infty} \frac{x^{k}}{k !} e^{-x} \frac{\Omega(d x)}{x}+\delta_{k, 1} b
$$

where

$\delta_{k, 1}=\left\{\begin{array}{l}\mathrm{I} \text { if } k=\mathrm{I} \\ \mathrm{O} \text { if } k \neq \mathrm{I}\end{array}\right.$

Proof

We have $E \exp \{i u N(t)\}=E \exp \left\{\Lambda(t)\left(e^{i u}-\mathbf{I}\right\}\right)$.

Since $\operatorname{Re}\left(e^{i u}-\mathrm{I}\right) \leqslant 0$ we have

$$
\int_{0+}^{\infty} \frac{e^{x\left(e^{i u}-I\right)}}{x} \Omega(d x)+b\left(e^{i u}-\mathrm{I}\right)=\sum_{k=1}^{\infty}\left(e^{i u k}-\mathrm{I}\right) c_{k}
$$

Thus the theorem holds since

$$
\begin{aligned}
& \left(e^{i u}-\mathrm{I}\right) b+\sum_{x=0}^{\infty} \int_{0+}^{\infty} \frac{\left(x e^{i u}\right)^{k}-x^{k}}{k !} e^{-x} \frac{\Omega(d x)}{x}= \\
& =\left(e^{i u}-\mathrm{I}\right) b+\int_{0+}^{\infty}\left(e^{x e^{i u}}-e^{x}\right) e^{-x} \frac{\Omega(d x)}{x}= \\
& =\left(e^{i u}-\mathrm{I}\right) b+\int_{0_{+}}^{\infty} \frac{e^{x\left(e^{i u}-\mathrm{I}\right)}}{x} \Omega(d x)
\end{aligned}
$$

because of dominated convergence since $e^{x}-\mathrm{I}$ is integrable with respect to $\frac{e^{-x} \Omega(d x)}{x}$. 


\section{Example}

Put $\Omega(d x)=\alpha e^{-\beta x} d x$ and $b=0$.

We have

$$
\eta(u)=\int_{0}^{\infty} \frac{e^{i x u}-\mathrm{I}}{x} \alpha e^{-\beta} x d x=-\alpha \log \left(\mathrm{I}-\frac{i u}{\beta}\right)
$$

and thus

$$
E e^{i u \Lambda(t)}=\left(\mathrm{I}-\frac{i u}{\beta}\right)^{-\alpha t}
$$

$\Lambda(t)$ is then $\Gamma$-distributed with the frequency function given by

$$
\frac{\beta^{\alpha t}}{\Gamma(\alpha t)} x^{(\alpha t-1)} e^{-\beta} x \text { for } x \geqslant 0
$$

By direct calculations we get

$$
E e^{i u_{N}(t)}=\int_{0}^{\infty} e^{x(e i u-1)} \frac{\beta^{\alpha t}}{\Gamma(\alpha t)} x^{(\alpha t-1)} e^{-\beta x} d x=\left(\frac{\beta}{\beta+\mathbf{I}-e^{i u}}\right)^{\alpha t}
$$

from which it follows that

$$
P(N(t)=k)=\left(\frac{\alpha t}{k}\right)\left(\frac{\beta}{\beta+\mathrm{I}}\right)^{\alpha t}\left(\frac{-\mathrm{I}}{\beta+\mathrm{I}}\right)^{k} \text { for } k=\mathrm{I}, 2, \ldots
$$

From the theorem it follows that

$$
c_{k}=\frac{\alpha}{k(\beta+\mathrm{I})^{k}} \text { for } k=\mathrm{I}, 2, \ldots
$$

Now the probability of ruin may be calculated in the following manner. Assume that $E \Lambda(t)=t$ which implies that $E N(t)=\mathrm{I}$ and that $v_{1}=E X_{k}$ exists. The probability of ruin $\psi\left(y_{0}\right)$ is then the probability that $y_{0}+\left(v_{1}+x\right) t-X(t)$ falls below zero at any time $t \geqslant 0$ where $y_{0}$ is the initial value of the risk reserve and $x$ the safety loading [2].

The bunches of claims will occur according to a Poisson process with intensity $c$ and the amounts to be paid for each bunch will form a sequence of independent random variables each having the distribution function

$$
\sum_{k=1}^{\infty} \frac{c_{k}}{c} \mathrm{~V}^{k *}(x)
$$

where $V^{k *}(x)$ is the $k$-fold convolution of $V(x)$ with itself. Define $x_{0}$ by 


$$
\left(v_{1}+x\right) t=\left(x_{0}+\sum_{k=1}^{\infty} \frac{c_{k}}{c} k v_{1}\right) c t
$$

from which it follows that

$$
x_{0}=\frac{v_{1}+x-v_{1} \sum_{k-1}^{\infty} c_{k} k}{c}
$$

Under the additional assumption that

$$
\sum_{k=1}^{\infty} c_{k} \int_{0}^{\infty} e^{\sigma x} \mathrm{~V} k *(d x)<\infty \text { for some } \sigma>0
$$

$\psi\left(y_{0}\right)$ may be calculated according to the derivation in Cramér [2] where $c t$ plays the role of the operational time, $x_{0}$ of the safety loading and $\sum_{k=1}^{\infty} \frac{c_{k}}{c} V^{k *}(x)$ of the distribution of the amount of each claim.

3. The CASE of a Directing PRocess with interchangeable INCREMENTS

The directing process $\{\Lambda(t) ; 0 \leqslant t<\infty\}$ is said to have interchangeable increments if for all $n=2,3, \ldots$ and all finite $T \varepsilon$ $(0, \infty)$ it holds that

$$
\begin{aligned}
& P\left[\bigcap_{k-1}^{n}\left\{\Lambda\left(\frac{k T}{n}\right)-\Lambda\left(\frac{(k-\mathrm{I}) T}{n}\right) \leqslant x_{k}\right\}\right]= \\
& =P\left[\bigcap_{k=1}^{n}\left\{\Lambda\left(\frac{k T}{n}\right)-\Lambda\left(\frac{(k-\mathrm{I}) T}{n}\right) \leqslant x_{i_{k}}\right\}\right]
\end{aligned}
$$

for all $n$ ! permutations $\left(i_{1}, \ldots, i_{n}\right)$ of $(\mathrm{I}, \ldots, n)$ and for all $\left(x_{1}, \ldots\right.$, $\left.x_{n}\right)$.

Bühlmann $[\mathbf{I}]$ has shown that for a process with interchangeable increments there exists a nontrivial sub- $\sigma$-algebra $\mathfrak{D}$ of $\mathfrak{l}$ such that $\Lambda(t)$ is a s.i.i. process relative to $\mathfrak{D}$. Further there exists a measure $\Pi_{\mathfrak{D}}$ on $\mathfrak{D}$ such that for every $0 \leqslant s<t<\infty$ we have

$$
E e^{i u(\Lambda(t)-\Lambda(s))}=\int e^{(t-s) \eta(u, \omega)} \Pi_{\mathfrak{D}}(d \omega)
$$

where

$$
\eta(u, \omega)=\int_{0+}^{\infty} \frac{e^{i u x}-\mathrm{I}}{x} \Omega(d x, \omega)+i b(\omega) u
$$

is $\mathfrak{D}$-measurable in $\omega$. 
Denote by $\Pi \mathfrak{D}_{\mathfrak{R}}$ the measure on $\mathfrak{R}$ relative to $\mathfrak{D}$ and by $\Pi \mathfrak{D}_{\mathfrak{L}}$ the measure on $\mathfrak{N}$ relative to $\mathfrak{D}$. Due to the theorem of Kolmogorov $\Pi \mathfrak{D}_{\mathfrak{R}}$ and thus $\Pi \mathfrak{D}_{\mathfrak{N}}$ are determined.

Since $\Lambda(t)$ is a s.i.i. process relative to $\mathfrak{D}$ also $N(t)$ is a.s i.i. process relative to $\mathfrak{D}$. Define

$$
c_{k}(\omega)=\int_{1+}^{\infty} \frac{x^{k}}{k !} e^{-x} \frac{\Omega(d x, \omega)}{x}+\delta_{k, 1} b(\omega)
$$

The sequence $\left\{c_{k}(\omega)\right\}_{k=1}^{\infty}$ is thus a sequence of $D$-measurable functions. From the theorem of Kolmogorov and from the theorem in section 2 it follows that the restriction $\Pi \mathfrak{D}_{\mathfrak{N}_{0}}$ of $\Pi \mathfrak{D}_{\mathfrak{N}}$ to $\mathfrak{N}_{0}$ may be expressed in terms of the sequence $\left\{c_{k}(\omega)\right\}_{k-1}^{\infty}$.

Assume that $E \Lambda(t)=t$. Define the $\mathfrak{D}$-measurable functions

$$
\begin{aligned}
& c(\omega)=\sum_{k=1}^{\infty} c_{k}(\omega) \\
& V(x, \omega)=\sum_{k=1}^{\infty} \frac{c_{k}(\omega)}{c(\omega)} V^{*}(x) \\
& v_{1}(\omega)=v_{1} \sum_{k=1}^{\infty} \frac{c_{k}(\omega)}{c(\omega)} k
\end{aligned}
$$

and

$$
\chi_{0}(\omega)=\frac{v_{1}(\omega)+x-v_{1} \sum_{k=1}^{\infty} c_{k}(\omega) \cdot k}{c(\omega)}
$$

If for almost every $\omega$ with respect to $\Pi_{D}$

$$
\int_{0}^{\infty} e^{\sigma x} V(d x, \omega)<\infty \text { for some } \sigma>0
$$

the probability of ruin $\psi^{\mathfrak{S}}\left(y_{0}\right)$ relative to $\mathfrak{D}$ may be calculated. Because of the assumption of separability the function $I(\omega)=\left\{\begin{array}{l}\mathrm{I} \text { if } y_{0}+\left(v_{1}+x\right) t-X(t)<0 \text { for some } t \geqslant 0 \\ 0 \text { elsewhere }\end{array}\right.$ is $\mathfrak{X}_{0}$-measurable and thus furthermore $\mathfrak{X}$-measurable. Since

$$
\psi \mathfrak{D}\left(y_{0}\right)=E(I(\omega) \mid \mathfrak{D})
$$

$\psi \mathfrak{D}\left(y_{0}\right)$ is $\mathfrak{D}$-measurable and $\psi\left(y_{0}\right)$ is given by

$$
\psi\left(y_{0}\right)=\int \psi \mathfrak{D}\left(y_{0}\right) d \Pi_{\mathfrak{D}}
$$




\section{Remark}

If $\Lambda(t)=\lambda \cdot t$ where $\lambda$ is a $\Gamma$-distributed random variable $N(t)$ is a Polya-process. In Segerdahl [4] the ruin probability is calculated by a weighting procedure. If $\Omega(d x, \omega)=0$ almost surely with respect to $\Pi_{\mathfrak{D}}$ and if $\lambda(\omega)=b(\omega)$ our result reduces to the result due to Segerdahl.

\section{A numerical illustration}

Consider the case where $N(t)$ is a Polya-process and where $V(x)= \begin{cases}\mathrm{I}-e^{-x} & \text { for } x \geqslant 0 \\ 0 & \text { for } x<0 .\end{cases}$

In our notations this implies that if $\lambda$ is a random variable defined on $(\Omega, \mathfrak{D}, \Pi)$ where

$$
\Pi_{\mathfrak{D}}(\lambda \leqslant x)=\int_{0}^{\infty} \frac{y^{h-1} h^{h}}{\Gamma(h)} e^{-h y} d y
$$

then $\Lambda(t)=\lambda \cdot t$ almost surely with respect to $\Pi \mathfrak{D}_{\mathfrak{R}}$.

In Cramér [2] it is shown that for this choice of $V(x)$ the ruin probability $\psi\left(y_{0}\right)$ is in the Poisson case (with intensity I) given by

$\psi\left(y_{0}\right)=\left\{\begin{array}{cc}\frac{I}{I+x} e^{-\frac{y_{0} x}{I+x}} \text { for } x>0 \\ I \quad \text { for } x \leqslant 0\end{array}\right.$

Since in the Polya case $v_{1}(\omega)=\mathrm{I}, c(\omega)=\lambda(\omega)$ and $c_{k}(\omega)=0$ for $k=2,3, \ldots$ almost surely with respect to $\Pi_{\mathfrak{D}}$ it follows that $x_{0}(\omega)=\frac{I+x_{0}-\lambda(\omega)}{\lambda(\omega)}$.

Thus

$$
\psi \mathcal{D}\left(y_{0}\right)= \begin{cases}\frac{\lambda(\omega)}{\mathrm{I}+x} e^{-\frac{\mathrm{I}+x-\lambda(\omega)}{\mathrm{I}+x} y_{0}} & \text { for } \lambda(\omega)<\mathrm{I}+x \\ \mathrm{I} & \text { for } \lambda(\omega) \geqslant \mathbf{I}+x\end{cases}
$$

Define the function

$$
\Gamma(x, \alpha, \beta)=\int_{0}^{x} \frac{y^{\alpha-1} \beta^{\alpha}}{\Gamma(\alpha)} e^{-\beta y} d y .
$$

It then follows that 


$$
\begin{gathered}
\psi\left(y_{0}\right)=\mathrm{I}-\Gamma(\mathrm{I}+x, h, h)+ \\
\left(\frac{h}{h-\frac{y_{0}}{\mathrm{I}+x}}\right)^{h+\mathrm{I}} \frac{e^{-y_{0}}}{\mathrm{I}+x} \Gamma\left(\mathrm{I}+x, h+\mathrm{I}, h-\frac{y_{0}}{\mathrm{I}+x}\right) .
\end{gathered}
$$

In tables $I, \ldots, 4$ this function is calculated for $x=0.0$, o.I, 0.2 and 0.3 and for $h=\mathrm{r}, 2,5$, 10, 25, 50 and roo. These values are further compared with the ruin probabilities in the Poisson case which is indicated by $h=\infty$.

\begin{tabular}{|c|c|c|c|c|c|c|c|c|}
\hline \multirow{2}{*}{$\frac{x=0}{y_{0}}$} & \multicolumn{8}{|c|}{$\psi\left(y_{0}\right)$} \\
\hline & $h=\mathrm{I}$ & $h=2$ & $h=5$ & $h=\mathrm{IO}$ & $h=25$ & $h=5^{\circ}$ & $h=\mathrm{IOO}$ & $h=\infty$ \\
\hline 0 & $0.632 \mathrm{I}$ & 0.7293 & 0.8245 & 0.8749 & 0.9205 & 0.9437 & $0.960 \mathrm{I}$ & I.0000 \\
\hline Io & 0.4042 & $0.45^{89}$ & 0.5236 & 0.5717 & 0.6380 & 0.6905 & 0.7429 & I.0000 \\
\hline 20 & $0.3^{862}$ & 0.4329 & $0.4^{8} 38$ & $0.5^{189}$ & $0.567 \mathrm{I}$ & 0.6079 & 0.6533 & 1.0000 \\
\hline 30 & $0.3^{80 \mathrm{r}}$ & $0.4^{2} 4^{\circ}$ & 0.4696 & 0.4992 & 0.5379 & 0.5703 & 0.6077 & 1.0000 \\
\hline $4^{\circ}$ & $0.377 \mathrm{I}$ & 0.4195 & 0.4624 & 0.4890 & 0.5223 & 0.5495 & 0.5809 & 1.0000 \\
\hline 50 & $0.375^{2}$ & $0.4 \mathrm{I} 68$ & 0.4580 & 0.4829 & 0.5128 & 0.5364 & 0.5635 & 1.0000 \\
\hline 60 & $0.374^{\circ}$ & $0.4^{\mathrm{I}} 5^{\circ}$ & $0.455 \mathrm{I}$ & 0.4787 & 0.5063 & 0.5275 & $0.55 \mathrm{I} 4$ & 1.0000 \\
\hline 70 & 0.3731 & 0.4137 & 0.4530 & $0.475^{8}$ & 0.5017 & 0.5210 & 0.5425 & 1.0000 \\
\hline 80 & 0.3725 & 0.4128 & $0.45 \mathrm{I} 5$ & 0.4735 & 0.4982 & $0.516 \mathrm{I}$ & $0.535^{8}$ & 1.0000 \\
\hline 90 & 0.3720 & $0.4 \mathrm{I} 20$ & 0.4502 & 0.4718 & 0.4954 & 0.5123 & 0.5305 & 1.0000 \\
\hline IOO & 0.3716 & $0.4 \mathrm{II} 4$ & 0.4493 & 0.4704 & 0.4932 & 0.5092 & 0.5262 & I.0000 \\
\hline$\rightarrow \infty$ & 0.3679 & 0.4060 & 0.4405 & 0.4579 & 0.4734 & $0.4^{812}$ & 0.4867 & 1.0000 \\
\hline
\end{tabular}

Table I

\begin{tabular}{|c|c|c|c|c|c|c|c|c|}
\hline $\begin{array}{l}x= \\
0.1\end{array}$ & \multicolumn{8}{|c|}{$\psi\left(y_{0}\right)$} \\
\hline$y_{0}$ & $h=\mathrm{I}$ & $h=2$ & $h=5$ & $h=\mathrm{ro}$ & $h=25$ & $h=5^{\circ}$ & $h=\mathbf{1 0 0}$ & $h=\infty$ \\
\hline 0 & 0.6065 & 0.6976 & $0.785^{8}$ & $0.83 \pm 5$ & $0.87 \mathrm{I} 3$ & 0.8897 & 0.9008 & 0.9091 \\
\hline Io & 0.3694 & 0.4080 & 0.4425 & 0.4588 & 0.4686 & 0.4663 & 0.4545 & 0.3663 \\
\hline 20 & $0.35^{12}$ & $0.3^{8} \mathrm{I}_{5}$ & 0.4009 & 0.4016 & $0.3^{847}$ & 0.3578 & 0.3186 & 0.1476 \\
\hline 30 & $0.345^{\mathrm{I}}$ & 0.3725 & 0.3864 & $0.3^{8} \mathrm{I} 2$ & 0.3533 & $0.3 \mathrm{I} 5 \mathrm{I}$ & 0.2626 & 0.0595 \\
\hline $4^{\circ}$ & 0.3420 & 0.3680 & 0.3791 & 0.3709 & 0.3373 & 0.2934 & 0.2340 & $0.024^{\circ}$ \\
\hline $5^{\circ}$ & 0.3402 & o.3653 & $0.374^{8}$ & 0.3648 & 0.3278 & 0.2806 & $0.2 \mathrm{I} 72$ & 0.0097 \\
\hline 60 & 0.3390 & 0.3635 & 0.3719 & 0.3607 & $0.32 \mathrm{I} 5$ & $0.272 \mathrm{I}$ & 0.2064 & 0.0039 \\
\hline 70 & $0.33^{8 \mathrm{I}}$ & 0.3623 & 0.3698 & 0.3578 & 0.3171 & $0.266 \mathrm{I}$ & O.I988 & 0.0016 \\
\hline 80 & 0.3375 & 0.3613 & 0.3683 & $0.355^{6}$ & 0.3137 & $0.26 \mathrm{I} 7$ & 0.1933 & 0.0006 \\
\hline 90 & 0.3369 & 0.3605 & $0.367 \mathrm{I}$ & 0.3539 & 0.3112 & 0.2583 & 0.1890 & 0.0003 \\
\hline IOO & $0.33^{65}$ & 0.3599 & $0.366 \mathrm{I}$ & $0.35^{26}$ & 0.3091 & $0.255^{6}$ & 0.1857 & 0.0001 \\
\hline$\rightarrow \infty$ & 0.3329 & 0.3546 & 0.3575 & 0.3405 & $0.29 \mathrm{IO}$ & 0.2322 & o.1584 & 0.0000 \\
\hline
\end{tabular}

Table 2 
Table 3

\begin{tabular}{|c|c|c|c|c|c|c|c|c|}
\hline$x=$ & \multicolumn{8}{|c|}{$\psi\left(y_{0}\right)$} \\
\hline$y_{0}$ & $h=\mathrm{I}$ & $h=2$ & $h=5$ & $h=$ Io & $h=25$ & $h=\mathbf{5}^{\circ}$ & $h=100$ & $h=\infty$ \\
\hline 0 & $0.5^{823}$ & $0.667^{\circ}$ & $0.747^{\circ}$ & 0.7864 & 0.8169 & 0.8280 & 0.8323 & 0.8333 \\
\hline ro & 0.3376 & $0.3^{6} 15$ & 0.3686 & 0.3564 & 0.3180 & 0.2755 & 0.2305 & o. I 574 \\
\hline 20 & 0.3194 & $0.335^{\circ}$ & 0.3267 & 0.2988 & 0.2344 & o.1709 & 0.1088 & 0.0297 \\
\hline 30 & 0.3133 & 0.3261 & 0.3126 & 0.2793 & 0.2064 & 0.1368 & $0.07 \mathrm{I} 7$ & 0.0056 \\
\hline $4^{\circ}$ & 0.3103 & $0.32 \times 6$ & $0.305^{6}$ & 0.2698 & o.1930 & 0.1213 & 0.0564 & 0.00 I I \\
\hline $5^{\circ}$ & 0.3084 & 0.3190 & 0.3014 & $0.264 \mathrm{I}$ & o. 1853 & o. I I 27 & 0.0486 & 0.0002 \\
\hline 60 & 0.3072 & 0.3 & 0.2987 & 0.2604 & 0.1803 & o.1073 & $0.044^{\circ}$ & 0.0000 \\
\hline $7^{\circ}$ & $0.306_{4}$ & $0.3^{\text {I } 59}$ & 0.2967 & $0.257^{8}$ & o.1 768 & 0.1036 & 0.04 I I & 0.0000 \\
\hline 80 & 0.3057 & 0.3150 & $0.295^{2}$ & $0.255^{8}$ & 0.1742 & 0.1009 & 0.0390 & 0.0000 \\
\hline 90 & 0.3052 & $0.3^{I} 43$ & $0.294 \mathrm{I}$ & 0.2543 & 0.1722 & 0.0989 & 0.0375 & 0.0000 \\
\hline roo & 0.3048 & $0.3^{1} 37$ & $0.293^{2}$ & $0.253^{\mathrm{I}}$ & 0.1707 & 0.0973 & 0.0363 & 0.0000 \\
\hline$\rightarrow$ & 0.3012 & 0.3084 & $0.285^{I}$ & 0.2424 & o. $x_{573}$ & 0.0844 & 0.0280 & 0.0000 \\
\hline
\end{tabular}

Table 4

\begin{tabular}{r|r|l|l|l|l|l|l|l}
\hline $\begin{array}{r}x= \\
0.3\end{array}$ & \multicolumn{7}{|c}{$\psi\left(y_{0}\right)$} \\
$y_{0}$ & $h=I$ & $h=2$ & $h=5$ & $h=I 0$ & $h=25$ & $h=50$ & $h=100$ & $h=\infty$ \\
\hline 0 & 0.5596 & 0.6378 & 0.7090 & 0.7414 & 0.7628 & $0.768 \mathrm{I}$ & 0.7692 & 0.7692 \\
10 & 0.3086 & 0.3194 & 0.3032 & $0.269 \mathrm{I}$ & 0.2026 & 0.1502 & $0.1 \mathrm{I} 22$ & 0.0765 \\
20 & 0.2905 & 0.2931 & 0.2624 & 0.2146 & 0.1297 & 0.0686 & 0.0304 & 0.0076 \\
30 & 0.2844 & 0.2844 & 0.2490 & 0.1972 & 0.1083 & 0.0477 & 0.0138 & 0.0008 \\
40 & 0.2815 & 0.2801 & 0.2425 & 0.1889 & 0.0987 & 0.0395 & 0.0088 & 0.0001 \\
50 & 0.2797 & 0.2775 & 0.2386 & 0.1840 & 0.0934 & 0.0353 & 0.0067 & 0.0000 \\
60 & 0.2785 & 0.2758 & 0.2361 & 0.1808 & 0.0900 & 0.0329 & 0.0056 & 0.0000 \\
70 & 0.2776 & 0.2766 & 0.2343 & 0.1786 & 0.0877 & 0.0312 & 0.0050 & 0.0000 \\
80 & 0.2770 & 0.2737 & 0.2329 & 0.1770 & 0.0860 & 0.0301 & 0.0046 & 0.0000 \\
90 & 0.2765 & 0.2730 & 0.2319 & 0.1757 & 0.0848 & 0.0292 & 0.0043 & 0.0000 \\
100 & 0.2761 & 0.2724 & $0.231 \mathrm{I}$ & 0.1747 & 0.0837 & 0.0286 & $0.004 \mathrm{I}$ & 0.0000 \\
$\rightarrow \infty$ & 0.2725 & 0.2674 & 0.2237 & 0.1658 & 0.0754 & 0.0236 & 0.0028 & 0.0000 \\
\hline
\end{tabular}

REFERENCES

[I] Bühlmann H., Austauschbare stochastische Variabeln und ihre Grenzwertsätze, Univ. of Cal. Publ. in Stat. 3, 1960.

[2] Cramér H., Collective risk theory, Jubilee volume of Försäkringsaktiebolaget Skandia, Stockholm, 1955.

[3] FELLER W., An introduction to probability theory and its applications Vol. II, John Wiley \& Sons, New York, I966.

[4] Segrerdahl C.-O., Lecture notes, University of Stockholm, I967.

[5] Walldin K.-E., Aspects of operations research in theory and practice, Almqvist \& Wiksell, Uppsala, I969. 